Pacific Journal of Mathematics

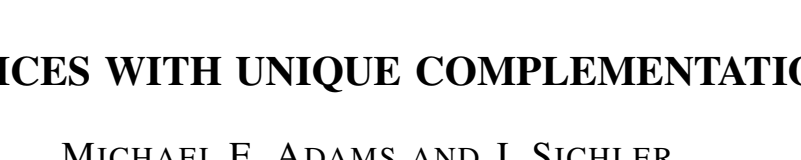




\title{
LATTICES WITH UNIQUE COMPLEMENTATION
}

\author{
M. E. AdAms AND J. SichleR
}

\begin{abstract}
R. P. Dilworth's theorem that every lattice is a sublattice of a uniquely complemented lattice is shown to hold in $2^{\boldsymbol{N}_{0}}$ varieties of lattices.
\end{abstract}

1. Introduction. After E. V. Huntington [11], it was conjectured that every uniquely complemented lattice was a distributive lattice; since a uniquely complemented distributive lattice is a Boolean lattice and every Boolean lattice is uniquely complemented, the verification of such a conjecture would have provided a characterization of Boolean lattices. For a uniquely complemented lattice $L$, G. Birkhoff and J. von Neumann showed that if $L$ is modular or relatively complemented then $L$ is distributive. Subsequently, G. Birkhoff and M. Ward [4] showed that if $L$ is complete, atomic, and dually atomic then $L$ is distributive. Further, R. P. Dilworth [6] verified that if $L$ is finite dimensional then it is distributive. Finally, the conjecture was refuted in, the now famous paper, [7]; R. P. Dilworth proved that every lattice is a sublattice of a uniquely complemented lattice (see also C. C. Chen and G. Grätzer [5]). Since then a number of other sufficient conditions for distributivity of a uniquely complemented lattice have been discovered. For example, if a uniquely complemented lattice $L$ is either atomic (T. Ogasawara and U. Sasaki [15], and J. E. McLaughlin [14]), algebraic (V. N. Salii [17]), or if the function that sends $l \in L$ to the unique complement of $l$ is order inverting (G. Birkhoff [3]), then $L$ is distributive.

The lattices constructed by R. P. Dilworth in [7] contain the free lattice on countably many generators as a sublattice. Hence, in particular, any nontrivial lattice identity fails to hold in any of Dilworth's lattices. (By a nontrivial identity, we mean an identity that does not follow from the lattice axioms.) A growing conjecture has been that any uniquely complemented lattice that satisfies a nontrivial lattice identity is distributive. In this connection (see G. Grätzer [9]), R. Padmanabhan [16] has shown that a uniquely complemented lattice in the variety $\boldsymbol{M} \vee \boldsymbol{N}_{5}$, or in the variety generated by a finite lattice satisfying one of two implications (namely, $\left(S D_{\wedge}\right)$ or an implication due to E. Fried and G. Grätzer, [9]) is distributive. However, we will show that this is not indicative of the general situation; that is to say, we will show that there are $2^{\aleph_{0}}$ varieties of lattices for which Dilworth's theorem holds. Thus, we will prove: 
THEOREM 1. There is a system of of $2^{\aleph_{0}}$ varieties of lattices such that, for $V \in \mathscr{Y}$, every $L \in V$ is a sublattice of a uniquely complemented lattice $L^{\prime} \in V$.

Clearly, Theorem 1 refutes the aforementioned conjecture. Choose a proper variety $V \in \mathscr{V}$ which is not the variety of distributive lattices. Let $L \in V$ be a nondistributive lattice. By Theorem 1 , the uniquely complemented lattice $L^{\prime}$ satisfies a nontrivial lattice identity (since $L^{\prime} \in V$ ) and is a nondistributive lattice (since $L$ is a sublattice of $L^{\prime}$ ).

In fact, rather than proving Theorem 1, we will prove the following stronger result:

THEOREM 2. There is a system of of $2^{\aleph_{0}}$ varieties of lattices such that, for $V \in \mathscr{Y}$, if $L \in V$ is a $(0,1)$-lattice, each element of which has at most one complement, then $L$ is a $(0,1)$-sublattice of a uniquely completed $(0,1)$-lattice $L^{\prime} \in V$.

Since, for example, G. Birkhoff and J. von Neumann have shown that every uniquely complemented modular lattice is distributive, it follows that not every lattice satisfying a nontrivial identity can be embedded in a uniquely complemented lattice that satisfies the same nontrivial identity. However, we will show that every lattice $L$ that satisfies a nontrivial identity is a sublattice of a uniquely complemented lattice $L^{\prime}$ that satisfies a (not necessarily the same) nontrivial identity. Furthermore, the construction presented is such that if $L$ is a locally finite lattice then $L^{\prime}$ is also a locally finite lattice. (A lattice is called locally finite if every finite subset generates a finite sublattice.) Thus, we prove:

THEOREM 3. Every lattice $L$ that satisfies a non-trivial identity $I$ is a sublattice of a uniquely complemented lattice $L^{\prime}$ that satisfies a (not necessarily the same) nontrivial identity $I^{\prime}$. Moreover, if $L$ is a locally finite lattice then $L^{\prime}$ may also be chosen to be a locally finite lattice.

We remark that, since the free lattice on three generators is infinite, none of Dilworth's lattices are locally finite. Moreover, every variety $V \in \mathscr{C}$ referred to above contains the variety of modular lattices as a subvariety and, as such, is not a locally finite variety. (A variety of lattices is locally finite if every lattice in the variety is locally finite.)

Once again we will, in fact, prove a stronger result: 
THEOREM 4. Let $L$ be a $(0,1)$-lattice each element of which has at most one complement. If $L$ satisfies a nontrivial identity $I$ then $L$ is a $(0,1)$-sublattice of a uniquely complemented $(0,1)$-lattice $L^{\prime}$ that satisfies a (not necessarily the same) nontrivial identity $I^{\prime}$. Furthermore, if $L$ is a locally finite lattice then $L^{\prime}$ may also be chosen to be a locally finite lattice.

We remark that the proofs of the above theorems yield an essentially new method of constructing nondistributive uniquely complemented lattices.

For all lattice theoretic terms not defined here the reader is referred to G. Grätzer [9]. (We observe that, in particular, Theorem 1 provides a solution to several of the research problems stated in [9].)

We would like to acknowledge fruitful discussions with G. Grätzer and to thank J. Berman and A. Day for their comments concerning our earlier version of this article.

2. Preliminaries. Let $\mathscr{L}$ denote the finite lattice of Figure 1 . Further, let $\mathscr{L}_{w}$ denote the partial lattice (see Figure 2) obtained from the lattice $\mathscr{L}$ by excluding its least element and its greatest element.

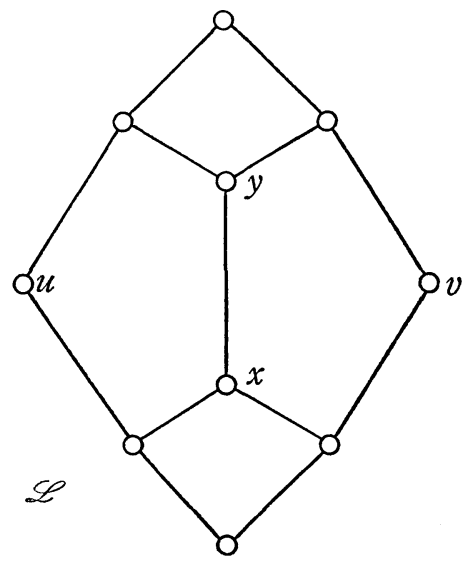

FiguRE 1

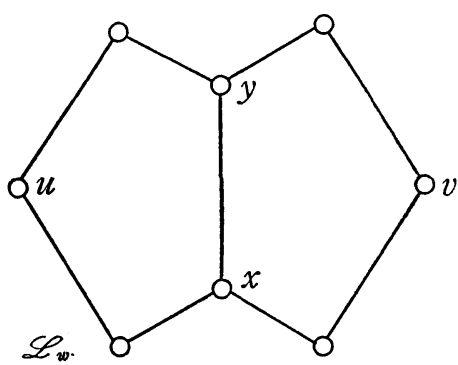

FIGURE 2

A bounded lattice is a lattice with a least element 0 and a greatest element 1. A bounded lattice in which the zero and unit elements are considered as distinguished elements is called a $(0,1)$ lattice.

For an arbitrary $(0,1)$-lattice $K, \mathscr{L}(K)$ denotes the lattice obtained by inserting the lattice $K$ in the interval $[x, y]$ of $\mathscr{L}$ (see Figure 3). Formally, let $\leqq$ be a relation defined on $\mathscr{L} \cup(K \backslash\{0,1\})$ 
by the following:

(i) For $a, b \in \mathscr{L}, a \leqq b$ if and only if $a \leqq b$ in $\mathscr{L}$,

(ii) For $a, b \in K \backslash\{0,1\}, a \leqq b$ if and only if $a \leqq b$ in $K$,

(iii) For $a \in K \backslash\{0,1\}, x<a<y$.

$\mathscr{L}(K)$ is the lattice whose order relation is the transitive closure of $\leqq$. Let $\mathscr{L}_{w}(K)$ denote the partial lattice obtained from the lattice $\mathscr{L}(K)$ by excluding its least element and its greatest element (see Figure 4). Observe that, in our notation, $\mathscr{L}$ and $\mathscr{L}_{w}$ are $\mathscr{L}(\mathbf{2})$ and $\mathscr{L}_{w}(2)$, respectively, where 2 denotes the two-element chain.

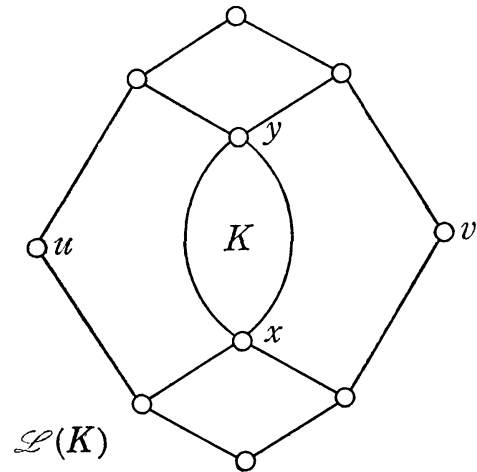

FiguRe 3

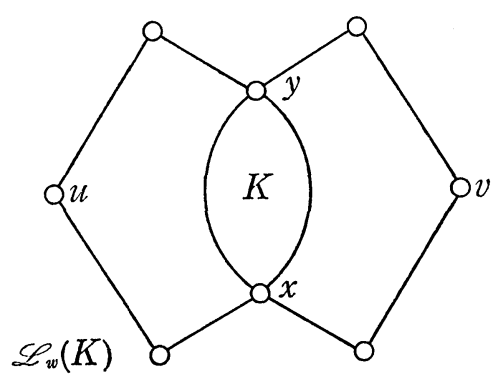

Figure 4

Lemma 5. If, for any $(0,1)$-lattice $K, \mathscr{L}_{w}(K)$ is a relative sublattice of a lattice $L$ then the sublattice of $L$ generated by $\mathscr{L}_{w}(K)$ is isomorphic to $\mathscr{L}(K)$.

Proof. The undefined joins of $\mathscr{L}_{w}(K)$ are $u \vee v, u \vee(y \vee v)$, $(u \vee y) \vee v$, and $(u \vee y) \vee(y \vee v)$. Observe that $u \vee v \leqq u \vee(y \vee v) \leqq$ $(u \vee y) \vee(y \vee v)$ and $u \vee v \leqq(u \vee y) \vee v \leqq(u \vee y) \vee(y \vee v)$ must hold in any lattice containing $\mathscr{L}_{w}(K)$. However, $u \vee v \geqq(u \wedge x) \vee(x \wedge v)=x$. Thus, $u \vee v=(u \vee x) \vee(x \vee v)=(u \vee y) \vee(y \vee v)$. Hence, the join of each pair of elements of $\mathscr{L}_{w}(K)$ that is undefined in equal to $(u \vee y) \vee(y \vee v)$, the largest element of the sublattice of $L$ generated by $\mathscr{L}_{w}(K)$. A dual argument shows that the meet of each pair of elements of $\mathscr{L}_{w}(K)$ that is undefined is equal to $(u \wedge x) \wedge$ $(x \wedge v)$, the smallest element of the sublattice generated by $\mathscr{L}_{w}(K)$ in $L$. It follows that the sublattice of $L$ generated by $\mathscr{L}_{w}(K)$ is isomorphic to $\mathscr{L}(K)$. Thus, the proof of Lemma 5 is complete.

Let $I$ be a lattice identity in $n$ variables. Then $I$ is of the form $\boldsymbol{p}\left(y_{0}, \cdots, y_{n-1}\right)=\boldsymbol{q}\left(y_{0}, \cdots, y_{n-1}\right)$, where $\boldsymbol{p}, \boldsymbol{q}$ are polynomial symbols and, for $i<n, y_{i}$ is a variable. For $i<n$, let

$$
\begin{aligned}
y_{i}^{\prime}= & \left(y_{i} \vee\left(x_{0} \wedge\left(x_{1} \vee x_{2}\right)\right) \vee\left(x_{1} \wedge\left(x_{0} \vee x_{2}\right)\right)\right) \\
& \wedge\left(x_{0} \vee\left(x_{1} \wedge\left(x_{0} \vee x_{2}\right)\right)\right) \wedge\left(x_{1} \vee\left(x_{0} \wedge\left(x_{1} \vee x_{2}\right)\right)\right) .
\end{aligned}
$$


$I^{\prime}$ will denote the lattice identity $\boldsymbol{p}\left(y_{0}^{\prime}, \cdots, y_{n-1}^{\prime}\right)=\boldsymbol{q}\left(y_{0}^{\prime}, \cdots, y_{n-1}^{\prime}\right)$. Observe that $I^{\prime}$ is an identity in $n+3$ variables (namely, $x_{0}, x_{1}, x_{2}$, and $y_{i}$, for $\left.i<n\right)$.

Before proceeding to the next lemma some motivation of the definition $I^{\prime}$ is in order.

For a lattice $L$, let $x_{0}, x_{1}, x_{2} \in L$. Eight elements of the lattice $L$ will be specified and then shown, under the assumption that a certain pair of them are distinct, to form a relative sublattice of $L$ isomorphic to $\mathscr{L}_{w}$. Consider the elements $x_{0}, x_{1}, x_{0} \wedge\left(x_{1} \vee x_{2}\right)$, $x_{1} \wedge\left(x_{0} \vee x_{2}\right), x_{0} \vee\left(x_{1} \wedge\left(x_{0} \vee x_{2}\right)\right), x_{1} \vee\left(x_{0} \wedge\left(x_{1} \vee x_{2}\right)\right),\left(x_{0} \wedge\left(x_{1} \vee x_{2}\right)\right) \vee$ $\left(x_{1} \wedge\left(x_{0} \vee x_{2}\right)\right)$, and $\left(x_{0} \vee\left(x_{1} \wedge\left(x_{0} \vee x_{2}\right)\right)\right) \wedge\left(x_{1} \vee\left(x_{0} \wedge\left(x_{1} \vee x_{2}\right)\right)\right)$. (It will be shown that these elements correspond to the elements $u, v$, $u \wedge x, x \wedge v, u \vee y, y \vee v, x$ and $y$ of $\mathscr{L}_{w}$, respectively.) Suppose that $\left(x_{0} \wedge\left(x_{1} \vee x_{2}\right)\right) \vee\left(x_{1} \wedge\left(x_{0} \vee x_{2}\right)\right)$ and $\left(x_{0} \vee\left(x_{1} \wedge\left(x_{0} \vee x_{2}\right)\right)\right) \wedge\left(x_{1} \vee\right.$ $\left.\left(x_{0} \wedge\left(x_{1} \vee x_{2}\right)\right)\right)$ are distinct elements of $L$ (see the definition of $I^{\prime}$ ). Certainly, $x_{0} \wedge\left(x_{1} \vee x_{2}\right) \leqq x_{0} \leqq x_{0} \vee\left(x_{1} \wedge\left(x_{0} \vee x_{2}\right)\right)$ and $x_{1} \wedge\left(x_{0} \vee x_{2}\right) \leqq$ $x_{1} \leqq x_{1} \vee\left(x_{0} \wedge\left(x_{1} \vee x_{2}\right)\right)$. Further, since $x_{0} \wedge\left(x_{1} \vee x_{2}\right) \leqq x_{1} \vee\left(x_{0} \wedge\right.$ $\left.\left(x_{1} \vee x_{2}\right)\right)$ and $x_{1} \wedge\left(x_{0} \vee x_{2}\right) \leqq x_{0} \vee\left(x_{1} \wedge\left(x_{0} \vee x_{2}\right)\right)$, it follows that $\left(x_{0} \wedge\left(x_{1} \vee x_{2}\right)\right) \vee\left(x_{1} \wedge\left(x_{0} \vee x_{2}\right)\right) \leqq\left(x_{0} \vee\left(x_{1} \wedge\left(x_{0} \vee x_{2}\right)\right)\right) \wedge\left(x_{1} \vee\left(x_{0} \wedge\right.\right.$ $\left.\left.\left(x_{1} \vee x_{2}\right)\right)\right)$. Hence, in order to show that the elements listed form a relative sublattice of $L$ isomorphic to $\mathscr{L}_{w}$ it is sufficient to show that the elements are distinct, the meet of $x_{0}$ and $x_{1} \vee\left(x_{0} \wedge\left(x_{1} \vee x_{2}\right)\right)$ is equal to $x_{0} \wedge\left(x_{1} \vee x_{2}\right)$, and, equivalently, the meet of $x_{1}$ and $x_{0} \vee$ $\left(x_{1} \wedge\left(x_{0} \vee x_{2}\right)\right)$ is equal to $x_{1} \wedge\left(x_{0} \vee x_{2}\right)$. Consider the meet of $x_{0}$ and $x_{1} \vee\left(x_{0} \wedge\left(x_{1} \vee x_{2}\right)\right)$. Clearly, $x_{0} \wedge\left(x_{1} \vee x_{2}\right) \leqq x_{1} \vee\left(x_{0} \wedge\left(x_{1} \vee x_{2}\right)\right)$ and, hence, $x_{0} \wedge\left(x_{1} \vee x_{2}\right) \leqq x_{0} \wedge\left(x_{1} \vee\left(x_{0} \wedge\left(x_{1} \vee x_{2}\right)\right)\right)$. Moreover, $x_{1} \vee$ $\left(x_{0} \wedge\left(x_{1} \vee x_{2}\right)\right) \leqq x_{1} \vee x_{2}$ and, whence, $x_{0} \wedge\left(x_{1} \vee\left(x_{0} \wedge\left(x_{1} \vee x_{2}\right)\right)\right) \leqq$ $x_{0} \wedge\left(x_{1} \vee x_{2}\right)$. Thus, $x_{0} \wedge\left(x_{1} \vee\left(x_{0} \wedge\left(x_{1} \vee x_{2}\right)\right)\right)=x_{0} \wedge\left(x_{1} \vee x_{2}\right)$; that is to say, the meet of $x_{0}$ and $x_{1} \vee\left(x_{0} \wedge\left(x_{1} \vee x_{2}\right)\right)$ is equal to $x_{0} \wedge$ $\left(x_{1} \vee x_{2}\right)$. It remains, therefore, to show that these eight elements are distinct; however, it is easily verified that if any two of the above elements are equal then it follows, contrary to assumption, that $\left(x_{0} \wedge\left(x_{1} \vee x_{2}\right)\right) \vee\left(x_{1} \wedge\left(x_{0} \vee x_{2}\right)\right)$ is equal to $\left(x_{0} \vee\left(x_{1} \wedge\left(x_{0} \vee x_{2}\right)\right)\right) \wedge$ $\left(x_{1} \vee\left(x_{0} \wedge\left(x_{1} \vee x_{2}\right)\right)\right)$. In conclusion, $L$ has a relative sublattice isomorphic to $\mathscr{L}_{w}$.

Lemma 6. Let $L$ be a lattice and $I$ a lattice identity. Then $L$ satisfies the lattice identity $I^{\prime}$ if and only if whenever $\mathscr{L}(K)$, for any bounded lattice $K$, is a sublattice of $L$ then $K$ satisfies the identity $I$.

Proof. Let $L$ be a lattice such that whenever $\mathscr{L}(K)$, for any bounded lattice $K$, is a sublattice of $L$ then $K$ satisfies the identity $I$. It will be shown that $L$ satisfies the identity $I^{\prime}$. 
Consider a substitution in $L$ for the variables $x_{0}, x_{1}, x_{2}$, and $y_{i}$, for $i<n$.

If the elements $\left(x_{0} \wedge\left(x_{1} \vee x_{2}\right)\right) \vee\left(x_{1} \wedge\left(x_{0} \vee x_{2}\right)\right)$ and $\left(x_{0} \vee\left(x_{1} \wedge\right.\right.$ $\left.\left.\left(x_{0} \vee x_{2}\right)\right)\right) \wedge\left(x_{1} \vee\left(x_{0} \wedge\left(x_{1} \vee x_{2}\right)\right)\right)$ are equal, then $y_{i}^{\prime}=y_{j}^{\prime}$ for all $i, j<n$. Hence, $\boldsymbol{p}\left(y_{0}^{\prime}, \cdots, y_{n-1}^{\prime}\right)=\boldsymbol{q}\left(y_{0}^{\prime}, \cdots, y_{n-1}^{\prime}\right)$.

Assume, therefore, that $\left(x_{0} \wedge\left(x_{1} \vee x_{2}\right)\right) \vee\left(x_{1} \wedge\left(x_{0} \vee x_{2}\right)\right)$ and $\left(x_{0} \vee\left(x_{1} \wedge\left(x_{0} \vee x_{2}\right)\right)\right) \wedge\left(x_{1} \vee\left(x_{0} \wedge\left(x_{1} \vee x_{2}\right)\right)\right)$ are distinct. By the remarks preceding the lemma, $L$ has a relative sublattice isomorphic to $\mathscr{L}_{w}$. Thus, by Lemma 5, there is an embedding $\psi: \mathscr{L} \rightarrow L$ such that $\psi(x)=\left(x_{0} \wedge\left(x_{1} \vee x_{2}\right)\right) \vee\left(x_{1} \wedge\left(x_{0} \vee x_{2}\right)\right)$ and $\psi(y)=\left(x_{0} \vee\left(x_{1} \wedge\right.\right.$ $\left.\left.\left(x_{0} \vee x_{2}\right)\right)\right) \wedge\left(x_{1} \vee\left(x_{0} \wedge\left(x_{1} \vee x_{2}\right)\right)\right)$. By definition, for $i<n, y_{i}^{\prime}$ is an element of the interval $[\psi(x), \psi(y)]$. By hypothesis, the interval $[\psi(x), \psi(y)]$ satisfies the identity $I$; that is to say, $\boldsymbol{p}\left(y_{0}^{\prime}, \cdots, y_{n-1}^{\prime}\right)=$ $\boldsymbol{q}\left(y_{0}^{\prime}, \cdots, y_{n-1}^{\prime}\right)$.

Either way, it follows that $L$ satisfies the identity $I^{\prime}$.

To prove the converse, suppose, to the contrary, that $L$ satisfies the identity $I^{\prime}$ and, for some bounded lattice $K$ that fails to satisfy the identity $I$, there exists an isomorphism $\psi: \mathscr{L}(K) \rightarrow L$. Thus, by assumption, there exist, for $i<n$, elements $y_{i}$ of the interval $[\psi(x)$, $\psi(y)]$ in $L$, such that $\boldsymbol{p}\left(y_{0}, \cdots, y_{n-1}\right) \neq \boldsymbol{q}\left(y_{0}, \cdots, y_{n-1}\right)$. Consider the substitution $\psi(u), \psi(v), \psi(y)$ for $x_{0}, x_{1}, x_{2}$, respectively. It is easily checked that $\psi(x)=\left(x_{0} \wedge\left(x_{1} \vee x_{2}\right)\right) \vee\left(x_{1} \wedge\left(x_{0} \vee x_{2}\right)\right)$ and $\psi(y)=\left(x_{0} \vee\right.$ $\left.\left(x_{1} \wedge\left(x_{0} \vee x_{2}\right)\right)\right) \wedge\left(x_{1} \vee\left(x_{0} \wedge\left(x_{1} \vee x_{2}\right)\right)\right)$. Hence, in this instance, $y_{i}=y_{i}^{\prime}$, for $i<n$. Thus, $\boldsymbol{p}\left(y_{0}^{\prime}, \cdots, y_{n-1}^{\prime}\right) \neq \boldsymbol{q}\left(y_{0}^{\prime}, \cdots, y_{n-1}^{\prime}\right)$; that is to say, since $L$ satisfies $I^{\prime}$, the above assumption leads to a contradiction. The proof of the lemma is complete.

A substantial part of the preceding lemma shows, in essence, that $\mathscr{L}$ is a finite subdirectly irreducible projective lattice; in other words, $\mathscr{L}$ is a splitting lattice. This is a known result due to $\mathrm{R}$. McKenzie [13] (see also B. Jónsson and J. B. Nation [12]). Since $\mathscr{L}$ is a splitting lattice the collection of those lattices $\mathscr{K}$ that do not contain $\mathscr{L}$ as a sublattice is closed under the operations $\boldsymbol{H}, \boldsymbol{S}, \boldsymbol{P}$. Thus, $\boldsymbol{H S P}(\mathscr{K})=\mathscr{K}$; that is to say, $\mathscr{K}$ is a variety. This variety has also been studied in E. Gedeonová [8], where such lattices are called $p$-modular. The conversion, presented here, of one identity to another is a generalization of a transformation (using the critical edge of a five element non-modular lattice) due to G. Grätzer and D. Kelly (unpublished)-see also R. McKenzie [13].

For a variety of lattices $V$ let $\mathscr{J}$ denote the collection of all those identities that are satisfied in $V$. Let $V^{\prime}$ denote the variety of lattices defined by the identities $\left\{I^{\prime}: I \in \mathscr{I}\right\}$.

LEMma 7. Let $L$ be a bounded lattice and $V$ a variety of lattices. Then $L \in V$ if and only if $\mathscr{L}(L) \in V^{\prime}$. 
Proof. If $L \notin V$ then there exists an identity $I \in \mathscr{J}$ such that $L$ does not satisfy $I$. That is to say, there exist, for $i<n, y_{i} \in L$ such that $\boldsymbol{p}\left(y_{0}, \cdots, y_{n-1}\right) \neq \boldsymbol{q}\left(y_{0}, \cdots, y_{n-1}\right)$. Consider the substitution $u, v, y$ for $x_{0}, x_{1}, x_{2}$, respectively, in $\mathscr{L}(L)$. It is easily seen that $\boldsymbol{p}\left(y_{0}^{\prime}, \cdots, y_{n-1}^{\prime}\right) \neq \boldsymbol{q}\left(y_{0}^{\prime}, \cdots, y_{n-1}^{\prime}\right)$; in other words, $\mathscr{L}(L)$ fails to satisfy the identity $I^{\prime}$. Hence, $\mathscr{L}(L) \notin V^{\prime}$.

It remains to show that $L \in V$ implies $\mathscr{L}(L) \in V^{\prime}$. Observe that, since $L \in V, L$ satisfies $I$, for all $I \in \mathscr{F}$. Consider the identity $I^{\prime}$, for some $I \in \mathscr{F}$. By Lemma $6, \mathscr{L}(L)$ satisfies $I^{\prime}$ if the existence of an embedding $\psi: \mathscr{L}(K) \rightarrow \mathscr{L}(L)$, for any bounded lattice $K$, always implies that $K$ satisfies the identity $I$. However, since $\psi$ is one-toone, both $\psi(x)$ and $\psi(y)$ are elements of $L$. Thus, $K$ is isomorphic to a sublattice of $L$ and, therefore, satisfies $I$. Hence, $\mathscr{L}(L)$ satisfies $I^{\prime}$, for all $I \in \mathscr{F}$; whence, $\mathscr{L}(L) \in V^{\prime}$. The proof is complete.

COROLlary 8. If lattice varieties $\boldsymbol{V}_{1}, \boldsymbol{V}_{2}$ are distinct, then $\boldsymbol{V}_{1}^{\prime}$, $V_{2}^{\prime}$ are distinct.

Proof. With no loss of generality, choose $L \in V_{1} \backslash V_{2}$. Since the lattice obtained by adding a new zero and a new unit to the lattice $L$ is also an element of $V_{1} \backslash V_{2}$, it may be assumed that $L$ is bounded. By Lemma 7, $\mathscr{L}(L) \in V_{1}^{\prime} \backslash \boldsymbol{V}_{2}^{\prime}$.

We now proceed to the main section of the paper. The constructions presented will be closely related to those in [1] and [2] (see also [10]); however, the proofs will be direct.

3. The main result. In order to deduce the theorems of $\S 1$, we will prove the following:

THEOREM 9. Let $L$ be a $(0,1)$-lattice for which each element has at most one complement. Then there exists a $(0,1)$-lattice $L^{+}$such that

(i) $L$ is isomorphic to a $(0,1)$-sublattice of $L^{+}$,

(ii) If $a \in L$ then a has a complement in $L^{+}$,

(iii) If $a \in L^{+}$then a has at most one complement in $L^{+}$,

(iv) For a lattice identity $I$, if $L$ satisfies $I^{\prime}$ then $L^{+}$satisfies $I^{\prime}$,

(v) If $L$ is locally finite then $L^{+}$is also locally finite.

We remark, as will become apparent from the construction, that if $L$ is a finite lattice then $L^{+}$will also be finite and if $L$ is an infinite lattice then $L$ and $L^{+}$will have the same cardinality.

To describe the lattice $L^{+}$associated with a given lattice $L$, some definitions and notation are necessary. 
Let $S \subseteq L$ be the set of all elements of $L$ with no complements in $L$. Clearly, $0 \notin S$ and thus $T=S \cup\{0\}$ is a disjoint union. For $s \in S$, let $L_{s}=C=\{0, x, 1\}$ be a three-element chain; set $L_{0}=L$ and consider the lower direct product $\Pi_{*}\left(L_{t}: t \in T\right)$, i.e., the sublattice of the direct product $\Pi\left(L_{t}: t \in T\right)$ of those sequences $p$ for which $p(t)=0$ for all but finitely many $t \in T$. Similarly, let $\Pi^{*}\left(L_{t}: t \in T\right)$ denote the upper weak direct product, in other words, the set of all elements $q \in \Pi\left(L_{t}: t \in T\right)$ such that $q(t)=1$ for all but finitely many $t \in T$.

For $s \in S$, let $p_{s} \in \Pi_{*}\left(L_{t}: t \in T\right)$ denote the element for which $p_{s}(0)=s, p_{s}(s)=x$, and $p_{s}(t)=0$ for all $t \in T \backslash\{0, s\}$; similarly, let $q_{s} \in \Pi^{*}\left(L_{t}: t \in T\right)$ be defined by $q_{s}(0)=s, q_{s}(s)=x$, and $q_{s}(t)=1$ for $t \in T \backslash\{0, s\}$.

Let $R_{*} \subseteq \Pi_{*}\left(L_{t}: t \in T\right)$ consist of all those $p$ for which at least one of the following holds:

(i) $p(t)=1$ for some $t \in T$,

(ii) $p \geqq p_{s}$ for some $s \in S$.

Similarly, let $R^{*} \subseteq \Pi^{*}\left(L_{t}: t \in T\right)$ be the set of all those sequences $q$ that satisfy one of the conditions below:

(i) $q(t)=0$ for some $t \in T$,

(ii) $q \leqq q_{s}$ for some $s \in S$.

Let $\Gamma_{*}$ denote the bounded lattice obtained by adding a new unit 1 to the poset $\Pi_{*}\left(L_{t}: t \in T\right) \backslash R_{*}$; the zero of $\Gamma_{*}$ is the constant sequence 0 defined by $\mathbf{0}(t)=0$ for all $t \in T$. Further, $\Gamma^{*}$ will denote the bounded lattice derived by adding a new zero 0 to $\Pi^{*}\left(L_{t}\right.$ : $t \in T) \backslash R^{*}$. The unit 1 of $\Gamma^{*}$ is the constant sequence with $1(t)=1$ for all $t \in T$. For $a \in L \backslash\{0,1\}$ define $\varphi_{*}(a) \in \Gamma_{*}$ as the sequence whose only nonzero value is $a=\left[\varphi_{*}(a)\right](0)$; set $\varphi_{*}(0)=\mathbf{0}$ and $\varphi_{*}(1)=\mathbf{1}$. If $s \in S$, let $s_{*} \in \Gamma_{*}$ be the sequence whose only nonzero value is $x=$ $s_{*}(s)$. Analogously, for $a \in L \backslash\{0,1\}$ let $\varphi^{*}(a) \in \Gamma^{*}$ be given by $\left[\varphi^{*}(a)\right](0)=a,\left[\varphi^{*}(a)\right](s)=1$ for all $s \in S$; set $\varphi^{*}(0)=0$ and $\varphi^{*}(1)=1$. Similarly to the above, $s^{*} \in \Gamma^{*}$ is the sequence defined by $s^{*}(s)=x$, $s^{*}(t)=1$ for $t \in T \backslash\{s\}$.

The lattice $L^{+}$can now be defined as the sublattice of $\Gamma=$ $\Gamma_{*} \times \Gamma^{*}$ generated by all pairs $\left(\varphi_{*}(a), \varphi^{*}(a)\right)$ for $a \in L$, by all elements $\left(s_{*}, s^{*}\right)$ with $s \in S$, and by $(0,1)$. It is easy to see that $(\mathbf{0}, 0)$ and $(\mathbf{1}, \mathbf{1})$ are the least and the largest element of $L^{+}$, respectively.

Clearly, $\varphi_{*}$ and $\varphi^{*}$ are one-to-one $(0,1)$-homomorphisms of $L$ into the lattices $\Gamma_{*}$ and $\Gamma^{*}$, respectively. The one-to-one homomorphism $\varphi: L \rightarrow L^{+}$defined by $\varphi(a)=\left(\varphi_{*}(a), \varphi^{*}(a)\right)$ yields the following claim.

Lemma $10 . \quad L$ is isomorphic to a $(0,1)$-sublattice of $L^{+}$. 
If $\{a, b\} \subseteq L$ is a complemented pair, then, by Lemma 10 , $\{\varphi(a), \varphi(b)\} \cong L^{+}$is a complemented pair as well. If $s \in L$ lacks a complement, then $s \in S$, and $\varphi_{*}(s) \vee s_{*}=1$ in $\Gamma_{*}$ since $\varphi_{*}(s) \vee s_{*} \geqq p_{s}$ holds in $\Pi_{*}\left(L_{t}: t \in T\right)$ and $p_{s} \in R_{*}$. Moreover, it is clear that $\varphi^{*}(s) \vee$ $s^{*}=1$ in $\Gamma^{*}$. Thus $\varphi(s) \vee\left(s_{*}, s^{*}\right)=\left(\varphi_{*}(s), \varphi^{*}(s)\right) \vee\left(s_{*}, s^{*}\right)=(1,1)$ in $L^{+}$. Similarly, $\varphi(s) \wedge\left(s_{*}, s^{*}\right)=\left(\varphi_{*}(s) \wedge s_{*}, \varphi^{*}(s) \wedge s^{*}\right)=(0,0)$ because $\varphi^{*}(s) \wedge s^{*} \leqq q_{s} \in R^{*}$. Hence $\varphi(s)$ has a complement $\left(s_{*}, s^{*}\right)$ in $L^{+}$. Where convenient, $L$ will be identified with its isomorphic copy determined by $\phi$ in $L^{+}$. Following is a consequence of these remarks.

Lemma 11. Every $a \in L$ has a complement in $L^{+}$.

It remains to be shown that all complemented pairs of $L^{+}$have been accounted for.

Lemma 12. If $(p, q) \in L^{+}$, then at least one of the following hold:

(i) $(p, q)=\left(\varphi_{*}(a), \varphi^{*}(b)\right)$ for some $a \leqq b$ in $L \backslash\{0,1\}$,

(ii) $(p, q)=\left(s_{*}, s^{*}\right)$ for some $s \in S$,

(iii) $p=0$ or $q=1$.

Proof. By definition, all generators of $L^{+}$satisfy the claim of the lemma. We proceed, therefore, inductively by the polynomial rank of an element of $L^{+}$; thus, we assume that $(p, q),(u, v) \in L^{+}$ satisfy the conclusion of the lemma and show that $(p, q) \wedge(u, v)$ satisfy one of the conditions (i), (ii), or (iii). The inductive step for $(p, q) \vee(u, v)$ is dual to that presented below and is, therefore, omitted.

Two observations are useful. First that, for every $u \in \Gamma_{*}$, $u \wedge s_{*}(a)>\mathbf{0}$ implies $u \geqq s_{*}$. Furthermore, if $u \in \Gamma_{*} \mid\{\mathbf{1}\}$, then $u \wedge$ $\varphi_{*}(a)>0$ yields $u(0) \wedge a>0$ in $L$; hence $u(0)=c \in L \backslash\{0\}$ and $u \wedge$ $\varphi_{*}(a)=\varphi_{*}(c \wedge a)$.

Let $(A, B)=(p, q) \wedge(u, v)$, and assume the lemma to be valid for $(p, q)$ and $(u, v)$. Thus, in particular, $u=1$ implies $v=1$ and the case $u=1$ need not be considered.

If $(p, q)=\left(\varphi_{*}(a), \varphi^{*}(b)\right)$ falls under (i), then $(A, B)$ satisfies (iii) unless $\varphi_{*}(a) \wedge u>\mathbf{0}$. It follows that $\varphi_{*}(a) \wedge u=\varphi_{*}(a \wedge c)$; if $v=\mathbf{1}$, then $(A, B)=\left(\varphi_{*}(a \wedge c), \varphi^{*}(b)\right)$ satisfies (i). If $v<1$, then, by the induction hypothesis, $(u, v)=\left(\varphi_{*}(c), \varphi^{*}(d)\right)$ satisfies the condition (i) and $(A, B)=\left(\varphi_{*}(a \wedge c), \varphi^{*}(b \wedge d)\right)$ is an element of type (i).

Secondly, let $(p, q)=\left(s_{*}, s^{*}\right)$ for some $s \in S$. If $s_{*} \wedge u=0$, then ( $A, B$ ) satisfies (iii); for $s_{*} \wedge u>0$ we obtain $u \geqq s_{*}$ and, by the induction hypothesis, $v=1$ or $(u, v)=\left(s_{*}, s^{*}\right)$. In either case, 
$(A, B)=\left(s_{*}, s^{*}\right)$ satisfies (ii).

If $p=0$, then $(A, B)=(0, q \wedge v)$ is described by (iii). Hence $q=1$ and $(A, B)=(p \wedge u, v)$ falls under (iii) unless $p \wedge u>0$ and $v<1$. By the inductive hypothesis, it follows that either $(u, v)=$ $\left(s_{*}, s^{*}\right)$ for some $s \in S$, or $(u, v)=\left(\varphi_{*}(a), \varphi^{*}(b)\right)$ must satisfy (i). The above arguments, applied to $(u, v)$ this time, complete the proof of the lemma.

Lemma 13. Every $(p, q) \in L^{+}$has at most one complement in $L^{+}$.

Proof. If $(u, v)$ is a complement of $(p, 1)$, then $p \wedge u=0$ and $v=\mathbf{0}$. Lemma 12 yields $u=\mathbf{0}$ and this, in turn, implies $p=1$. Arguing dually for $(0, q)$, we conclude that $(0,0)$ and $(1,1)$ form the only complemented pair of $L^{+}$involving at least one element satisfying (iii). From now on, we may assume that $u \in \Gamma_{*} \mid\{0,1\}$ and $v \in$ $\Gamma^{*} \backslash\{\mathbf{0}, \mathbf{1}\}$.

Let $(u, v)$ be a complement of $(p, q)=\left(s_{*}, s^{*}\right)$ for $s \in S$. Observe that $u \wedge s_{*}=0$ implies $u(s)=0$; hence $\left(u \vee s_{*}\right)(s)=x$, and $\left(u \vee s_{*}\right)(t)=u(t)$ for $t \in T \backslash\{s\}$. Since $u \vee s_{*}=\mathbf{1}$, the sequence $u \vee s_{*}$, considered as an element of $\Pi_{*}\left(L_{t}: t \in T\right)$, belongs to $R_{*}$. Hence $u \vee s_{*} \geqq p_{t}$ for some $t \in S$, since $\left(u \vee s_{*}\right)(t)<1$ for all $t \in T$. If $t \neq s$, then $u(0)=\left(u \vee s_{*}\right)(0) \geqq t$ and $u(t)=\left(u \vee s_{*}\right)(t) \geqq x$ and these imply $u \geqq p_{t}$ in $\Pi_{*}\left(L_{t}: t \in T\right)$; thus $u=1$ in $\Gamma_{*}-$ a contradiction. Hence $u \vee s_{*} \geqq p_{s}$ and, in particular, $1>u(0)=a \geqq s>0$ in $L$. By Lemma $12,(u, v)=\left(\varphi_{*}(a), \varphi^{*}(b)\right)$ for some $b \geqq a$ in $L$. On the other hand, $\varphi^{*}(b) \wedge s^{*}=0$ yields $\varphi^{*}(b) \wedge s^{*} \leqq q_{s}$ in $\Pi^{*}\left(L_{t}: t \in T\right)$; that is, $b=\left(\varphi^{*}(b) \wedge s^{*}\right)(0) \leqq q_{s}(0)=s$. Altogether, $a=b=s$; thus $\varphi(s)=$ $\left(\varphi_{*}(s), \varphi^{*}(s)\right)$ is the only complement of $\left(s_{*}, s^{*}\right)$ in $L^{+}$.

Let $(u, v)$ be a complement of $(p, q)=\left(\varphi_{*}(a), \varphi^{*}(b)\right)$ with $a \leqq b$ in $L \backslash\{0,1\}$. If $(u, v)=\left(\varphi_{*}(c), \varphi^{*}(d)\right)$ and $c \leqq d$, then $\{a, c\}$ and $\{b, d\}$ are complemented pairs of $L$. However, $a \leqq b$ and $c \leqq d$ imply that $\{b, c\}$ and $\{a, d\}$ are complemented pairs. Since every element of $L$ has at most one complement, we conclude $a=b, c=d$. Thus $(u, v)=\varphi(c)$ and $(p, q)=\varphi(a)$, where $c$ is the unique complement of $a$ in $L$. If $(u, v)=\left(s_{*}, s^{*}\right)$, for some $s \in S$, is a complement of $(p, q)$ then-as shown above- $(p, q)=\varphi(s)$; in particular, $(p, q)$ has no complements in $L$. By Lemma 13 and from the previous arguments it follows that all complements of $\varphi(s)$ must satisfy (ii); thus, any such complement is the form $\left(t_{*}, t^{*}\right)$ for some $t \in S$. However, since $\varphi(t)$ is the only complement of $\left(t_{*}, t^{*}\right)$ satisfying (i), we conclude that $s=t$. Thus every element of type (i) has at most one complement in $L^{+}$.

Lemma 14. Let $K$ be a bounded lattice. If $\mathscr{L}(K)$ is isomorphic 
to a sublattice of $\Gamma_{*}$ or $\Gamma^{*}$, then $\mathscr{L}(K)$ is isomorphic to a sublattice of $L$.

Proof. Suppose that $\mathscr{L}(K)$ is isomorphic to a sublattice of $\Gamma_{*}$. Recall that $\Gamma_{*}=\left(\Pi_{*}\left(L_{t}: t \in T\right) \backslash R_{*}\right) \cup\{1\}$; hence, $\mathscr{L}_{w}(K)$ is isomorphic to a relative sublattice of $\Pi_{*}\left(L_{t}: t \in T\right)$. By Lemma $5, \mathscr{L}(K)$ is isomorphic to a sublattice of $\Pi_{*}\left(L_{t}: t \in T\right)$. For $s \in S, L_{s}$ is a threeelement chain. Whence, the subdirectly irreducible lattice $\mathscr{L}$ is isomorphic to a sublattice of $L_{0}=L$. In particular, $x(0) \neq y(0)$ and, for $s \in S, x(s)=y(s)$. Thus, for $p, q \in \mathscr{L}, p(0) \neq q(0)$. Furthermore, it follows that if $p, q \in K$, then $p(s)=q(s)$ for all $s \in S$; hence $p(0) \neq$ $q(0)$ and $\mathscr{L}(K)$ is isomorphic to a sublattice of $L_{0}=L$. A dual argument shows that if $\mathscr{L}(K)$ is isomorphic to a sublattice of $\Gamma^{*}$ then $\mathscr{L}(K)$ is isomorphic to a sublattice of $L_{0}=L$.

Lemma 15. For a lattice identity $I$, if $L$ satisfies $I^{\prime}$ then $L^{+}$ also satisfies $I^{\prime}$.

Proof. Let $L$ satisfy the identity $I^{\prime}$. Since $L^{+}$is a sublattice of $\Gamma$, it is sufficient to show that $\Gamma_{*}$ and $\Gamma^{*}$ satisfy the identity $I^{\prime}$. By Lemma $6, \Gamma_{*}$ satisfies $I^{\prime}$ if whenever, for any bounded lattice $K$, $\mathscr{L}(K)$ is a sublattice of $\Gamma_{*}$ then $K$ satisfies the identity $I$. By Lemma 14 , if $\mathscr{L}(K)$ is a sublattice of $\Gamma_{*}$ then $\mathscr{L}(K)$ is isomorphic to a sublattice of $L$. Since $L$ satisfies $I^{\prime}$, it follows, again by Lemma 6 , that $K$ satisfies $I$. Hence, $\Gamma_{*}$ satisfies $I^{\prime}$. A dual argument shows that $\Gamma^{*}$ also satisfies the identity $I^{\prime}$.

LEMMA 16. If $L$ is locally finite then $L^{+}$is locally finite.

Proof. Let $L$ be a locally finite lattice. Since the direct product of two locally finite lattices is locally finite, in order to show that $L^{+}$is locally finite it is sufficient to show that $\Gamma_{*}$ and $\Gamma^{*}$ are locally finite. Choose a finite subset of $\Gamma_{*}=\left(\Pi_{*}\left(L_{t}: t \in T\right) \backslash R_{*}\right) \cup\{\mathbf{1}\}$. Clearly, the sublattice generated is a subset of the sublattice of $\Pi_{*}\left(L_{t}: t \in T\right)$ generated by those elements together with the element 1 . Since $L_{0}=L$ is a locally finite lattice and, for $s \in S, L_{s}$ is a three-element chain, the sublattice generated in $\Pi_{*}\left(L_{t}: t \in T\right)$ is finite. Thus, $\Gamma_{*}$ is locally finite; by a dual argument, $\Gamma^{*}$ is also locally finite.

Lemmas $10,11,13,15$, and 16 combine to prove Theorem 9.

4. The proof of Theorems 2 and 4 . Let $L$ be a $(0,1)$-lattice, each element of which has at most one complement. By Theorem $9, L$ is a $(0,1)$-sublattice of a lattice $L^{+}$such that every element of $L$ has a complement in $L^{+}$, yet every element of $L^{+}$has at most 
one complement. Let $L_{0}=L$ and inductively define, for $n<\omega$, $L_{n+1}=\left(L_{n}\right)^{+}$. Let $L^{\prime}=U\left(L_{n}: n \in \omega\right)$. Clearly, $L$ is a $(0,1)$-sublattice of $L^{\prime}$, and $L^{\prime}$ is uniquely complemented.

Let $\mathscr{V}$ denote the set of all those varieties $V^{\prime}$ associated with a lattice variety $V$. Let $V^{\prime} \in \mathscr{V}$. Then $L \in V^{\prime}$ if and only if $L$ satisfies $I^{\prime}$ for every identity $I$ holding in $V$. By Theorem 9, if $L$ satisfies $I^{\prime}$ then $L^{+}$satisfies $I^{\prime}$. Therefore, arguing inductively for $n<\omega, L_{n}$ also satisfies $I^{\prime}$. Since any finite subset of $L^{\prime}$ is contained in $L_{N}$ for some $N<\omega$, it follows that $L^{\prime}$ also satisfies the identity $I^{\prime}$. Thus, for $W \in \mathscr{Y}$, if $L \in W$ then $L^{\prime} \in W$. Furthermore, since there are $2^{\aleph_{0}}$ varieties of lattices it follows, by Corollary 8, that the cardinality of $\mathscr{V}$ is $2^{\aleph_{0}}$. The proof of Theorem 2 is complete. We remark that $\mathscr{V}$ has a smallest element: the variety associated with the splitting lattice $\mathscr{L}$.

Let $L$ satisfy a nontrivial identity $I$. Then, by Lemma $6, L$ also satisfies the identity $I^{\prime}$. By Corollary $8, I^{\prime}$ is also a non-trivial identity. Thus, arguing as above, $L^{\prime}$ also satisfies the nontrivial lattice identity $I^{\prime}$. Moreover, if $L$ is locally finite it follows, by Theorem 9 , that $L_{n}$ is locally finite, for $n<\omega$. Observe, once more, that any finite subset of $L^{\prime}$ is contained in $L_{N}$ for some $N<\omega$. Hence, $L^{\prime}$ is locally finite. Whence, Theorem 4 has been verified.

5. Concluding remarks. A number of questions arise naturally from the above. The most obvious one, although possibly rather ridiculous question, is to ask for a characterization of those varieties $V$ for which Theorem 1 is valid. A more realistic problem is to ask whether there exists a locally finite variety for which Theorem 1 holds.

In conclusion, it should be pointed out that Theorems 2 and 4 can be generalized (see C. C. Chen and G. Grätzer [5]), using the method presented here to the following:

THEOREM 17. There exist $2^{\aleph_{0}}$ varieties of lattices of such that, for $V \in \mathscr{Y}$ and every cardinal $\alpha \geqq 1$, if $L \in V$ is a $(0,1)$-lattice, each element of which has $\leqq \alpha$ distinct complements, there is a lattice $L_{\alpha}^{\prime} \in V$ containing $L$ as a $(0,1)$-sublattice and such that every element of $L_{\alpha}^{\prime}$ has exactly $\alpha$ distinct complements.

THEOREM 18. Let L be a $(0,1)$-lattice each element of which has $\leqq \alpha$ distinct complements, where $\alpha$ is a cardinal such that $\alpha \geqq 1$. If $L$ satisfies a nontrivial identity $I$ then $L$ is a $(0,1)$-sublattice of a lattice $L_{\alpha}^{\prime}$ such that every element of $L_{\alpha}^{\prime}$ has exactly $\alpha$ distinct complements and $L_{\alpha}^{\prime}$ satisfies a (not necessarily the same) nontrivial lattice identity $I^{\prime}$. 


\section{REFERENCES}

1. M. E. Adams and J. Sichler, Bounded endomorphisms of lattices of finite height, Cannad. J. Math., 29 (1977), 1254-1263.

2. - Cover set lattices, Canad. J. Math.

3. G. Birkhoff, Lattice theory (2 nd Edition), Amer. Math. Soc., Providence, R. I., 1948.

4. G. Birkhoff and M. Ward, A characterization of Boolean algebras, Ann. of Math., 40 (1939), 609-610.

5. C. C. Chen and G. Grätzer, On the construction of complemented lattices, J. Algebra, 11 (1969), 56-63.

6. R. P. Dilworth, On complemented lattices, Tôhoku Math. J., 47 (1940), 18-23.

7. - Lattices with unique complements, Trans. Amer. Math. Soc., 57 (1945), 123-154.

8. E. Gedeonová, Jordan-Hölder theorem for lines, Mat. Časopis Sloven. Akad. Vied., 22 (1972), 177-198.

9. G. Grätzer, General Lattice Theory, Birkhäuser Verlag, Basel and Stuttgart, 1978.

10. G. Grätzer and J. Sichler, On the endomorphism semigroup (and category) of bounded lattices, Pacific J. Math., 35 (1970), 639-647.

11. E. V. Huntington, Sets of independent postulates for the algebra of logic, Trans. Amer. Math. Soc., 5 (1904), 288-309.

12. B. Jónsson and J. B. Nation, A report on sublattices of a free lattice, Universal Algebra (Proc. Colloq. Szeged (1975)), Colloq. Math. Soc. János Bolyai, 17 (1977), 223-257.

13. R. McKenzie, Equational bases and non-modular lattice varieties, Trans. Amer. Math. Soc., 174 (1972), 1-43.

14. J. E. McLaughlin, Atomic lattices with unique comparable complements, Proc. Amer. Math. Soc., 7 (1956), 864-866.

15. T. Ogasawara and U. Sasaki, On a theorem in lattice theory, J. Sci. Hiroshima Univ. Ser. A, 14 (1949), 13.

16. R. Padmanabhan, Some non-modular varieties in which all uniquely complemented lattices are distributive, to appear.

17. V. N. Salī, A compactly generated lattice with unique complements is distributive (Russian), Mat. Zametki, 12 (1972), 617-620.

Received March 5, 1979. The support of the National Research Council of Canada is gratefully acknowledged.

UNIVERSiTy OF MANitoba,

Winnipeg, Manitoba, Canada. 



\section{PACIFIC JOURNAL OF MATHEMATICS}

\section{EDITORS}

DONALD BABBITT (Managing Editor)

University of Galifornia

Los Angeles, California 90024

Hugo RossI

University of Utah

Salt Lake City, UT 84112

C. C. MOORE AND ANDREW OGG

University of California

Berkeley, CA 94720
J. DUGUNDJI

Department of Mathematics University of Southern California Los Angeles, California 90007

R. Finn AND J. Milgram Stanford University Stanford, California 94305

\section{ASSOCIATE EDITORS}

R. ARENS

E. F. BECKENBACH

B. H. NEUManN

F. WOLF

K. YOSHIDA

\section{SUPPORTING INSTITUTIONS}

UNIVERSITY OF ARIZONA

UNIVERSITY OF BRITISH COLUMBIA CALIFORNIA INSTITUTE OF TECHNOLOGY

UNIVERSITY OF CALIFORNIA

MONTANA STATE UNIVERSITY

UNIVERSITY OF NEVADA, RENO

NEW MEXICO STATE UNIVERSITY

OREGON STATE UNIVERSITY
UNIVERSITY OF OREGON

UNIVERSITY OF SOUTHERN CALIFONIA

STANFORD UNIVERSITY

UNIVERSITY OF HAWAII

UNIVERSITY OF TOKYO

UNIVERSITY OF UTAH

WASHINGTON STATE UNIVERSITY

UNIVERSITY OF WASHINGTON 


\section{Pacific Journal of Mathematics}

\section{Vol. 92, No. $1 \quad$ January, 1981}

Michael E. Adams and J. Sichler, Lattices with unique complementation . ....1

Walter Allegretto, Positive solutions and spectral properties of second order

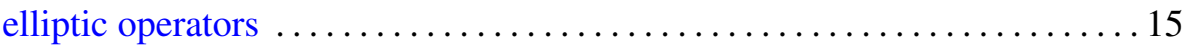

Philip J. Boland and Sean Dineen, Holomorphy on spaces of distribution . . 27

Duncan Alan Buell, Philip A. Leonard and Kenneth S. Williams, Note on

the quadratic character of a quadratic unit $\ldots \ldots \ldots \ldots \ldots \ldots \ldots \ldots \ldots$

Herbert Busemann and Bhalchandra B. Phadke, Two theorems on

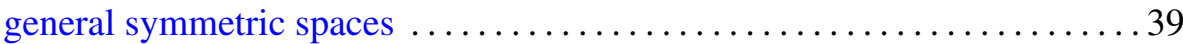

Emeric Deutsch, Bounds for the Perron root of a nonnegative irreducible

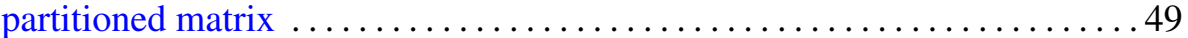

Charles F. Dunkl, A difference equation and Hahn polynomials in two

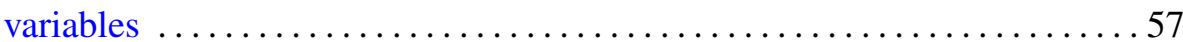

Gustave Adam Efroymson, The Riemann mapping theorem for planar Nash

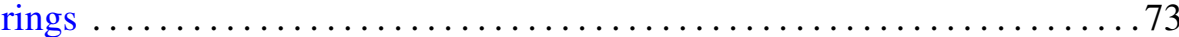

John Albert Fridy and Robert Ellis Powell, Tauberian theorems for matrices generated by analytic functions

Denton Elwood Hewgill, John Hamilton Reeder and Marvin Shinbrot,

Some exact solutions of the nonlinear problem of water waves .......887

Bessie Hershberger Kirkwood and Bernard Robert McDonald, The

symplectic group over a ring with one in its stable range

Esther Portnoy, Transitive groups of isometries on $H^{n}$

Jerry Ridenhour, On the sign of Green's functions for multipoint boundary

value problems

Nina M. Roy, An $M$-ideal characterization of $G$-spaces

Edward Barry Saff and Richard Steven Varga, On incomplete

polynomials. II

Takeyoshi Satō, The equations $\Delta u=P u(P \geqq 0)$ on Riemann surfaces and

isomorphisms between relative Hardy spaces

James Henry Schmerl, Correction to: "Peano models with many generic classes"

Charles Madison Stanton, On the closed ideals in $A(W)$

Viakalathur Shankar Sunder, Unitary equivalence to integral operators

Pavel G. Todorov, New explicit formulas for the $n$th derivative of composite functions

James Li-Ming Wang, Approximation by rational modules on boundary sets

Kenneth S. Williams, The class number of $Q(\sqrt{p})$ modulo 4 , for $p \equiv 5$

$(\bmod 8)$ a prime 